\title{
CMet CAR-mRNA Electroporated Autologous T Lymphocytes
}

\author{
National Cancer Institute
}

\section{Source}

National Cancer Institute. cMet CAR-mRNA Electroporated Autologous T Lymphocytes. NCI Thesaurus. Code C106230.

A preparation of autologous T-lymphocytes that have been electroporated with an mRNA encoding a chimeric antigen receptor (CAR) consisting of an anti-human hepatocyte growth factor receptor (HGFR or cMet) scFv (single chain variable fragment) and the zeta chain of the TCR/CD3 complex (CD3-zeta) coupled to the co-stimulatory molecule 4-1BB (CD137), with potential antineoplastic activities. Upon intratumoral administration, cMet CAR-mRNA electroporated autologous T lymphocytes direct T-cells to cMet-expressing tumor cells, which induces a selective toxicity in cMet-expressing tumor cells and causes tumor cell lysis. The 4-1BB co-stimulatory molecule signaling domain enhances activation and signaling after recognition of cMet. The inclusion of the 4-1BB signaling domain may increase the antitumor activity as compared to the inclusion of the CD3-zeta chain alone. The mRNA CAR is expressed for a limited amount of time, which can prevent serious, unforeseen side effects. cMet, a receptor tyrosine kinase overexpressed or mutated in many tumor cell types, plays a key role in cancer cell growth, survival, angiogenesis, invasion, and metastasis. 